\section{APUNTES CRÍTICOS SOBRE LAS DINÁMICAS DE INSTITUCIONALIZACIÓN DE LA INTERSECCIONALIDAD}

\author{
UXue Zugaza GoienetXeA \\ Universidad del País Vasco \\ uxue.zugaza@ehu.eus \\ ORCID iD: https://orcid.org/0000-0001-7613-9218
}

Cómo citar este artículo I Citation: Zugaza Goienetxea, U. 2020. "Apuntes críticos sobre las dinámicas de institucionalización de la interseccionalidad". Revista Internacional de Sociología 78(1): e152. https:// doi.org/10.3989/ris.2020.78.1.18.145

\begin{abstract}
Resumen
Esta nota pone en relación la institucionalización de la interseccionalidad y el progresivo desapego de las trayectorias activistas e intelectuales de las mujeres de color que la inspiraron. El texto sugiere que esta pérdida de radicalidad es la consecuencia lógica de cuatro dinámicas de institucionalización de la interseccionalidad, que son: 1) el olvido de la genealogía de color y el ethos de la justicia social, 2) un desplazamiento epistemológico hacia la disciplina y la estandarización, 3) la hegemonía de las producciones científicas del Norte global frente al Sur global y 4) la política de la innovación y los mercados de la diversidad en un contexto de gobernanza neoliberal.
\end{abstract}

\section{Palabras Clave}

Epistemología; Feminismos negros; Justicia social;

\section{CRITICAL NOTES ABOUT THE INSTITUTIONALIZATION OF INTERSECTIONALY}

Copyright: () 2020 CSIC. Este es un artículo de acceso abierto distribuido bajo los términos de la licencia de uso y distribución Creative Commons Reconocimiento 4.0 Internacional (CC BY 4.0).

Recibido: 28/09/2018. Aceptado: 17/07/2019

Publicado online: 16/03/2020

\section{Abstract}

This paper relates the institutionalization of intersectionality and the critics about the lack of its genealogy inspired by the intellectual and activist trajectories of women of color. The text suggests that the loose of radicalness responds to four dynamics of institutionalization of intersectionality. These are: 1) the forget of the genealogy of colour and the ethos of social justice, 2) an epistemological shift to the discipline and standardization, 3) the hegemony of the global North's scientific productions as opposed to those of global South and 4) the politics of innovation and the diversity market within a neoliberal context.

\section{KeYWORDS}

Black feminism; Epistemology; Neoliberalism; Social justice. 


\section{INTRODUCCIÓN}

Desde que la jurista afroamericana Kimberlé Crenshaw (1989) pusiera nombre a las formas complejas de experimentar la intersección entre el racismo y el sexismo hacia las mujeres negras en EEUU, han sido numerosas las aportaciones académicas que han abordado la interseccionalidad como un revulsivo para la profundización democrática (Martínez-Palacios y Martínez 2017). A las contribuciones normativas y metodológicas desde la teoría política feminista (McCall 2005; Yuval-Davis 2006, Hancock 2007; Walby 2007) le siguen trabajos que exploran las posibilidades de esta herramienta compleja en el diseño de políticas, poniendo el foco en los factores que inciden en la entrada de la interseccionalidad en la policy making (Krizsan, Skjeie y Squires 2012; Hankivsky y Cormier 2011; Kantola y Nousiainen 2009). Mientras esto ocurre, algunas posiciones críticas apuntan hacia el éxito de la interseccionalidad como el logro de una versión aséptica y neutralizada de una perspectiva cada vez más alejada de las críticas antirracistas que, desde el feminismo negro estadounidense, la inspiraron.

En el centro de esta última cuestión, aparecen dos obras decididas a reinyectar de dosis crítica a una herramienta cuyo potencial insurgente, advierten las autoras, parece haberse difuminado a lo largo de sus múltiples viajes y desarrollos: Intersectionality, de Patricia Hill Collins y Silma Bilge y L'intersectionnalité: enjeux théoriques et politiques, bajo la coordinación de Marta Roca i Escoda, Farinaz Fassa y Eléonore Lépinard, publicadas en el año 2016. Hilvanados desde diferentes contextos lingüísticos, intelectuales, activistas y políticos, pero unidos en un compromiso común con la justicia social, ambos proyectos exploran aspectos y desafíos teóricos y prácticos que enfrenta en la actualidad las dispersiones de la interseccionalidad. La progresiva asimilación de esta herramienta a través del marco discursivo de las políticas de igualdad en el Estado español (Platero 2012) y la lógica experto-burocrática que subyace en la gobernanza de la igualdad (Squires 2007) justifican la importancia de una lectura crítica en torno a la tensión entre institucionalizar y democratizar.

Ello porque la institucionalización, o la implementación de un conjunto "más o menos coordinado de reglas y procedimientos que gobiernan las interacciones de los actores y las organizaciones" (Lascoumes y Le Gales 2007: 8) ocurre a través de unos instrumentos que estructuran valores, creencias y principios de comportamiento anclados en matrices cognitivas y normativas establecidas (2007: 8). Si bien pudiera interpretarse como una palanca para revestir de legitimidad a las demandas de los movimientos sociales y un instrumento para su realización, la institucionalización de las ideas y los movimientos ocurre en unas estructuras que, de entrada, no presuponen una sensibilidad ética y ontológica hacia la inclusión democrática y la justicia social (Collins 2016). Por eso, y de la mano de las dos obras mencionadas, esta nota de investigación pone en relación la institucionalización de la interseccionalidad con la desactivación de los reclamos por la justicia social que laten en su genealogía.

A tal fin, a la revisión de las dos citadas obras le sigue un segundo apartado que desarrolla cuatro dinámicas que se desprenden de la crítica de ambos trabajos a la institucionalización de la interseccionalidad: 1) el olvido de la genealogía de color y el ethos de la justicia social, 2) un desplazamiento epistemológico hacia la disciplina y la estandarización de la interseccionalidad, 3) la hegemonía de las producciones científicas del Norte global frente al Sur global y 4) la política de la innovación y los mercados de la diversidad en un contexto de gobernanza neoliberal. La nota concluye con un apartado que pone en diálogo estas dinámicas con algunas ideas que se extraen de los primeros análisis de instrumentos de política locales y europeos para la implementación de la igualdad de género.

\section{DOS OBRAS EN TORNO A LOS DESAFíos TEÓRICOS Y PRÁCTICOS DE LA INTERSECCIO- NALIDAD}

El año 2012 tuvo lugar en Laussanne (Suiza) el VI Congreso Internacional de Investigaciones Feministas Francófonas. Bajo el título "Imbricación de las relaciones de poder: discriminaciones y privilegios de género, raza, clase y sexualidad", este congreso fue, además de un punto de encuentro de diferentes perspectivas feministas sensibles al cruce de desigualdades, un pretexto afortunado que animó la elaboración de las dos obras que componen esta nota de investigación.

Fruto del diálogo y del trabajo conjunto entre las sensibilidades de Patricia Hill Collins, desde el contexto estadounidense, y Sirma Bilge desde el contexto canadiense, Intersectionality es el resultado de la labor crítica para reorientar y reorganizar las múltiples aristas que entroncan con la idea de la interseccionalidad. Se trata, además, de una labor necesaria para reinyectar de radicalidad a un término que, según las autoras, la propia producción académica ha vuelto escurridizo y confuso.

El libro abre en su primer capítulo con una pregunta ("What is intersectionality?", pp. 1-30) que ya es muestra de todos los vaivenes conceptuales que ha suscitado el término. A la cuestión de qué es la interseccionalidad, las autoras responden definiendo la interseccionalidad como una herramienta analítica que puede tomar diferentes formas. La alusión a la interseccionalidad como herramienta supone, de entrada, un portazo a todos los debates conceptuales 
en torno a qué es o dónde encasillarla. De este modo, y a partir del análisis de tres fenómenos globales a la luz de la interseccionalidad, Collins y Bilge desvelan los core themes o ideas clave que componen esta herramienta, el esqueleto o la razón de ser de la interseccionalidad: la sensibilidad por la desigualdad, las relaciones de poder y el compromiso con la justicia social, el carácter relacional, la importancia del contexto social y la complejidad.

Las autoras dan continuidad a estos primeros trazos en el segundo capítulo (pp. 31-62), y profundizan en la idea de la interseccionalidad como una relación sinérgica entre la indagación crítica y la práctica crítica. Esta visión productiva entre la teoría y la práctica se enmarca en una estrategia analítica que desafía la artificial división intelectual-activista, traspasando las fronteras conceptuales que la encasillan como un método de investigación.

El tercer capítulo (pp. 63-87) rescata de los "olvidos" del saber institucionalizado la genealogía invisibilizada de la interseccionalidad. Las autoras elaboran una crítica muy oportuna en torno a la historia autorizada de la interseccionalidad, una visión lineal que descubre en el mítico texto de Kimberlé Crenshaw (1989) el punto de origen del concepto, oscureciendo la sinergia teoría-práctica propia de la interseccionalidad y presente a lo largo de las trayectorias y luchas políticas no narradas que, desde el activismo, la literatura o el arte han encarnado las mujeres racializadas.

A partir de esta visión retrospectiva, el cuarto capítulo (pp. 88-113) se ubica en la narración del panorama actual. Así, las veinticinco páginas que lo componen son testigo de la dispersión y el desarrollo global de la interseccionalidad en la actualidad hacia las instituciones, la academia y la red, a través del diálogo acerca de la interseccionalidad producido en estos espacios y los usos, debates y malentendidos que ha generado.

Muestra de una de las derivas de la interseccionalidad son los encuentros y desencuentros que han suscitado sus diálogos con las políticas de identidad, desarrollados en el quinto capítulo (pp. 114-135). Las autoras reivindican la memoria para no caer en malentendidos y evocan el clásico manifiesto de las Combahee River Collective (1981[1977]), en que la identidad, abrazada a un análisis estructural de las opresiones, constituye un revulsivo para la acción política. El sexto capítulo (pp. 136-158) ubica la interseccionalidad en un escenario transnacional y de movimientos globales de protesta frente al neoliberalismo, abordándola como una herramienta analítica para analizar la complejidad de los conflictos sociales en un contexto de capitalismo global.

El séptimo capítulo (pp. 159-190) elabora un sugerente diálogo entre la interseccionalidad y la pedagogía crítica de Paulo Freire. Ambos exploran puntos comunes que beben de preceptos anclados en la filosofía de la democracia participativa. El último capítulo (pp. 191-204) retoma conjuntamente todas las dimensiones abordadas en la obra a la luz de la "tensión creativa" entre la teoría crítica y la práctica crítica como un espacio productivo para pensar la interseccionalidad.

Desde el contexto francés, Marta Roca i Escoda, Farinaz Fassa y Eléonore Lépinard coordinan L'intersectionalité: enjeux théoriques et politiques. Los ecos del congreso de Laussanne marcan el pulso de esta obra colectiva. Ello ya es muestra de la proximidad y de las posibilidades de diálogo entre este trabajo y el recién expuesto de Collins y Bilge, motivado, como ya se ha dicho, por las reflexiones que surgieron a tenor de este mismo evento. Los nueve capítulos que conforman la obra se dividen en dos partes temáticas: la primera parte reflexiona en torno a las posibilidades contrahegemónicas de esta herramienta ante el "riesgo de diluir su potencial político en la retórica académica” (p. 16). La segunda parte analiza empíricamente la puesta en práctica de la interseccionalidad: el uso, las apropiaciones y los significados que toma en diferentes contextos.

Motivadas por la necesidad de recuperar de la interseccionalidad el espíritu contrahegemónico e insurgente que inspiró el activismo de las mujeres de color', las editoras reivindican en la introducción (pp. 7-26) la genealogía crítica y el alcance heurístico de la interseccionalidad.

Kimberlé Crenshaw, jurista afroamericana a la que se le ha otorgado la autoría del concepto, es la encargada de abrir, metafóricamente tal vez, el primer capítulo de la obra (pp. 29-51). A lo largo del texto la autora muestra su perplejidad ante las interpretaciones, viajes y debates posteriores al "bautismo" de la interseccionalidad. Tal y como ella la entiende, la interseccionalidad no es ni una teoría universal, ni una palabra de moda, ni un concepto al que se pueda disciplinar. Muy al contrario, la autora reivindica la utilidad práctica con la que concibió la interseccionalidad en unos trabajos inspirados en el Black Feminism y la tradición jurídica crítica; dos impulsos fundamentales que la popularización del término a través de las teorías mainstream en Europa ha ido borrando progresivamente.

En la misma línea, Patricia Hill Collins expone en el segundo capítulo (pp. 53-74) la necesidad de recuperar de la idea de interseccionalidad el corazón que lo hace latir: la justicia social. La necesidad de mantener viva esta pulsión crítica no es sino una respuesta inevitable a la genealogía activista y de color de la que germinó el pensamiento interseccional. La asimilación de la interseccionalidad en los códigos del campo académico del Norte global en un contexto de individualismo y excelencia, alerta la autora, ha desactivado el ethos orientado a la justicia social. 
Sirma Bilge firma el tercer capítulo (pp. 75-102), haciendo una crítica audaz al "blanqueamiento" de la interseccionalidad que, en un contexto de excelencia y estandarización, pilota la nueva industria universitaria. Frente al descentramiento de la raza y la neutralización del carácter indudablemente político que orientó las críticas de las mujeres de color, la canadiense reivindica la interseccionalidad como una praxis utopista que sitúe el antirracismo radical y la crítica al neoliberalismo en el centro de su reflexión. En el cuarto capítulo (pp. 103-129), Cornelia Möser analiza los diferentes relatos a los que ha dado lugar el uso del concepto género en el contexto del feminismo académico. La autora sitúa esta evolución discursiva en un escenario inquietante de transformación del modelo universitario en relación con la adaptación a las demandas del mercado y su influencia en el pensamiento crítico.

La segunda parte del libro comienza con el quinto capítulo, de Erica Townsend-Bell (pp. 133-158), en el que la autora indaga en torno a las coaliciones entre grupos feministas en Uruguay como una forma de praxis interseccional. Éléonore Lépinard continúa explorando las posibilidades de la praxis interseccional en el sexto capítulo (pp. 159-188). Con el objetivo de analizar el impacto de las cuotas de género en el contexto de la representación política a la luz de la interseccionalidad, concluye de manera interesante que el modelo universalista del republicanismo francés y una herencia fuerte del feminismo materialista han bloqueado una perspectiva interseccional de la igualdad de género. En el séptimo capítulo (pp. 189-221), Raluca Popa y Andrea Krizsan analizan factores que explican la praxis interseccional en las alianzas y las coaliciones entre grupos activistas movilizados en la lucha contra la violencia hacia las mujeres, a partir de los casos de Hungría y Rumanía.

Marilène Lieber explora, en el octavo capítulo (pp. 223-247), el uso heurístico de la interseccionalidad para problematizar los sesgos racistas y de clase a que ha dado lugar la construcción del problema del acoso sexual callejero en las ciudades y su influencia en el diseño de las mal llamadas "ciudades inclusivas". Finalmente, el libro (noveno capítulo, pp. 249-277) concluye con un texto de Lucia Direnberger que analiza, a través de la construcción discursiva de "la mujer musulmana", los posos orientalistas y colonialistas en el escenario postsoviético de Tayikistán.

Tras este breve recorrido, el siguiente punto extrae de estas dos obras varias dinámicas de institucionalización de la interseccionalidad para ponerlas en diálogo con algunos síntomas de desradicalización que acusa. Ello ayudará a comprender y reorientar las dimensiones que comprometen el sentido crítico de la interseccionalidad.

\section{Comprender la pérdida de Radicali- DAD DE LA INTERSECCIONALIDAD A TRAVÉS DE SUS DINÁMICAS DE INSTITUCIONALIZACIÓN}

\section{EI "olvido" de la genealogía de color y el ethos de la justicia social}

El primer síntoma de este "mal", la pérdida de radicalidad de la interseccionalidad, mira hacia el olvido de dos dimensiones fundamentales en la práctica y crítica interseccional: la dimensión racial y el compromiso con la justicia social. Los "malos viajes" (Martínez-Palacios y Martínez 2017: 11) y las dispersiones de la interseccionalidad han acabado por silenciar la riqueza de las trayectorias activistas e intelectuales de los feminismos de color que inspiraron las luchas por la justicia social y el fin de las opresiones que las atravesaban. La desactivación de la razón crítica de la interseccionalidad a través de un alejamiento progresivo del compromiso con la justicia social que, desde una perspectiva estructuralista, hilvanaron las diferentes voces del feminismo negro es, sin duda, un punto nodal de todas las sensibilidades críticas que analizan el desarrollo y la institucionalización de esta herramienta.

A este respecto, el olvido de la dimensión racial no es baladí. En este punto, es oportuno traer el asombro de Crenshaw frente al debate suscitado en torno a la inclusión de la raza en los estudios interseccionales. Un debate que, dirá, evoca la vieja idea de que la presencia de las mujeres negras compromete la representatividad del universal (Crenshaw, 1989), y que lleva implícito el planteamiento de que los estudios de raza suponen una subdisciplina aparte, incapaz de trascender su particularidad y, por tanto, no son aptos para elaborar teorías "universalmente consumibles" (Crenshaw 2016: 34).

La omisión de la raza va íntimamente ligada a las "prácticas de citación" o "las políticas de localización" (Bilge 2016: 94) que preconizan revistas científicas, cursos, conferencias y manuales que canonizan a algunos textos y autoras, estableciendo fronteras de conocimiento raciales y discursivas. Estas prácticas no son sino la materialización misma de las omisiones, resistencias y modificaciones de un campo discursivo de poder y dominación que circunda el mundo académico (Crenshaw 2016: 33). Además de no hacer justicia a la centralidad de las mujeres de color en tanto que productoras legítimas de "saberes interseccionales" (Collins 2000), este desplazamiento retrata un feminismo en perfecta armonía respecto al feminismo hegemónico, que borra de un plumazo las tensiones y las interpelaciones que, desde las luchas antirracistas y a favor de la justicia social, han producido las mujeres de color (Bilge 2016: 92-3). 


\section{Un desplazamiento epistemológico hacia la disciplina y la estandarización de la intersec- cionalidad}

En segundo lugar, la popularización de la interseccionalidad camina en paralelo a las tendencias de adaptación de los saberes a unos criterios estandarizados de validez y cientificidad que rigen el campo del saber institucionalizado (Crenshaw 2016; Bilge 2016; Collins 2016). La necesidad de encasillar la interseccionalidad como una teoría general capaz de anticipar las desigualdades, sus dinámicas y las estructuras y categorías que la componen responde a una ansiedad por estandarizar, medir y clasificar toda producción de conocimiento (Crenshaw 2016: 34). En un contexto de "sed de cientificidad" promovido por marcos positivistas (Bilge 2016: 89), la interseccionalidad se ve abocada a demostrar su madurez científica y sus posibilidades de aplicación para resolver conflictos y problemas sociales concretos.

Se trata de "domesticar" un concepto rebelde a través de una verdadera "industria de autentificación" (Crenshaw 2016: 48). El precio de la legitimidad en el marco del saber institucionalizado pasa, tal y como explica Collins, por la asimilación de ciertos códigos para transformar la interseccionalidad en un concepto reconocible y comprensible desde los parámetros académicos de autoridad y propiedad intelectual (2016: 61). Tal es el caso de atribuir autorías de conceptos que, por otro lado, parecen ser "descubiertos" en el momento en que un agente bien posicionado en el campo académico los enuncia y los reviste de legitimidad.

De este modo, tras una primera "fase rudimentaria" donde las mujeres negras elaboran conocimientos particulares y especializados (Bilge 2016: 89), el rito de acceso de la interseccionalidad al campo académico pasa por la conversión a un paradigma de conocimiento general que produzca consenso en el seno de las diferentes disciplinas de conocimiento.

\section{Hegemonía de las producciones científicas del Norte global frente al Sur global}

A través de la omisión de la crítica racial y poscolonial y de las formas recién enunciadas de disciplinarla, pareciera que la interseccionalidad es el nuevo continente que el colonizador "descubre", aquella tierra desconocida que no existía previa a su llegada (Bilge 2016: 99). Ello nos traslada a la tercera dinámica de institucionalización que analiza, desde un punto de vista crítico, esta nota de investigación.

En perfecta consonancia con las ideologías colonialistas, la producción y la recepción de los saberes se distribuye a través de las fronteras geopolíticas entre el Norte y el Sur globales. El desplazamiento de la genealogía activista y el "habitus racializado" de la interseccionalidad se acompaña, pues, de una política de localización de la interseccionalidad en que la he- gemonía de autoras del Norte global eclipsa por completo las producciones del Sur global (Bilge 2016). Este es un fenómeno conocido en el ámbito de la democracia participativa a través del caso de los presupuestos participativos (Baiocchi y Ganuza 2017).

Esta frontera de reconocimiento Norte-Sur no solo orienta la importación de conceptos que provienen de países como EEUU o Europa, sino también la omisión de realidades y experiencias ubicadas en las propias narrativas locales (Bilge 2016: 95). Existe toda una serie de "dispositivos de control", en el sentido foucaultiano del término (Collins 2016: 73), orientados a estimular unos cánones determinados de excelencia e internacionalización que se enmarcan en esta división. Tal es el caso, por ejemplo, de los estándares internacionales de calidad que establecen la idoneidad de una investigación para su financiación (Möser 2016: 124-5), y que invitan a problematizar los códigos que estimulan y bloquean determinadas iniciativas de intervención e investigación.

Inserta en esta división Norte-Sur, la estandarización que se mencionaba en el apartado anterior se materializa por medio de los impulsos de armonización de las políticas, a través de directivas de organismos supranacionales que se trasponen y se aplican en el ámbito doméstico de los Estados nación (Möser 2016: 116). La implementación de políticas top-down a través de directivas y trasposiciones de políticas interseccionales en el ámbito de la Unión Europea (UE) son un buen ejemplo de estas dinámicas (Verloo 2006; Kantola y Nousiainen 2009; Lombardo y Verloo 2010). En este punto, resulta oportuno reflexionar en torno a la relación entre las lógicas de implementación top-down (en detrimento de las políticas bottom-up), la omisión de la riqueza del terreno en lo local y la hegemonía clara del Norte global en la producción y en el diseño de los saberes que se materializan en políticas.

\section{La política de la innovación y los mercados de la diversidad en un contexto de gober- nanza neoliberal}

Las interacciones entre el conocimiento y las necesidades de una ciencia positivista orientada a las demandas del mercado han sido una constante a lo largo de las reflexiones y conforman el cuarto punto que cierra este apartado: la innovación como discurso y estrategia política que estimula, por otro lado, la creación de un mercado específico para cubrir esa demanda.

La crítica se enmarca en un contexto inquietante, donde el pensamiento crítico y sosegado es golpeado por fuertes corrientes utilitaristas que animan la conversión de las "industrias" universitarias. Este cambio de modelo vendría gobernado, tal y como dice Bilge, por lógicas neoliberales de producción, profesionalización elitista, superespecialización y conocimiento experto; una evidente jerarquización de las disciplinas 
en función de su utilidad económica y una fijación con el rigor metodológico, acompañado, como se ha visto, por una creciente desactivación del activismo en el seno de la academia (Bilge 2016: 101). Al compás de las "economías temporales" (Möser 2016: 212), que rigen un paradigma científico desbordado por la inmediatez, las ideas "desactualizadas" se descartan y se las reviste de un glamour que exige su actualización. La cultura del control, orientada por lógicas neoliberales de excelencia y logro individual -a través de dispositivos de evaluación, promoción, etc.- - se ubica en claro antagonismo al ethos colectivo que promovían los movimientos sociales e intelectuales que inspiraron la praxis interseccional (Collins 2016: 73).

El lenguaje de la innovación y los criterios de utilidad serían testigos de lo que Möser denomina la "crisis general de la ciencia" (Möser 2016: 121) y que evidencian diversos ejemplos. Es el caso de la retórica de la innovación de la Nueva Gestión Pública, que evoca la entrada de este cambio de paradigma en las administraciones públicas. Siguiendo con Möser, el reclamo de la experticia - a través de técnicas y técnicos - y la puesta en horizonte de resultados estandarizados y cuantificables, generan cuestiones de suma importancia en relación con la institucionalización de herramientas guiadas por la política de la innovación. En un contexto gobernado por el espíritu de la novedad, no sorprende la conversión de herramientas inicialmente inspiradas en la transformación y la justicia social en cebos para la obtención de fuentes de financiación (Möser 2016).

Otro ejemplo ilustrativo es la progresión peligrosa que describen Collins y Bilge hacia una idea de la diversidad como "buena práctica" a llevar a cabo por las instituciones y las universidades (Collins y Bilge 2016: 185). Alejado de las aguas comprometidas con la democracia inclusiva, las autoras hablan de un planteamiento de la diversidad que vira hacia el terreno de las competencias culturales en su versión "benigna y purificada" (Bilge 2016: 88). Para ello, dicen, se despliega una auténtica maquinaria dirigida a la adquisición de dichas competencias - consultorías, workshops, materiales audiovisuales, etc.-. Las autoras hablan de un "mercado de la diversidad", donde esta pasa a ser un atractivo para revalorizar las propias instituciones (Collins y Bilge 2016: 186).

\section{EL RETO DE UNA INSTITUCIONALIZACIÓN CRÍTICA DE LA INTERSECCIONALIDAD}

Leídas a la luz de la crítica al enfoque experto-burocrático de las políticas de igualdad que preconiza la Nueva Gestión Pública (Squires 2007), se intuye cierta complicidad entre estas dinámicas y la omisión de las voces outsiders (Collins 2000) que emerge a través de los procesos de institucionalización de la igualdad de género. Del análisis de veintiséis planes locales de igualdad vigentes en las capitales de provincia del Estado español y seis documentos estratégicos de la UE en este ámbito, se desprenden algunas ideas para pensar estas dinámicas como dispositivos que limitan la entrada de una sensibilidad y una praxis interseccional (Collins y Bilge 2016) en estas políticas.

Si bien los planes son ricos en referencias a las estructuras de desigualdad que, en cruce con el género, organizan las relaciones de opresión y privilegio entre las mujeres, ello ocurre, en algunos casos, a través de la atribución de roles estigmatizantes y pasivos de lasmujeres condicionadas por desigualdades en cruce, y la asimilación de la interseccionalidad a través de estas políticas apenas se acompaña de referencias al racismo y las estructuras de raza. La alusión al crecimiento económico, por otro lado, como principal argumento para la promoción de la igualdad entre mujeres y hombres en algunos municipios, constituye un síntoma evidente de la pérdida de marcos de justicia social en el discurso de la desigualdad de género. Ciertamente, esto último toma su reflejo en el discurso pro igualdad de la UE, actor central en las referencias normativas que citan los planes locales y agente clave en la popularización de las discriminaciones múltiples en la política comunitaria (Lombardo y Verloo 2010), del que sobresale, en el contexto de los documentos analizados, un enfoque orientado al crecimiento económico y la promoción laboral de las mujeres como ámbitos prioritarios de actuación de las políticas de igualdad. En este sentido, se identifica la estandarización como parte clave del impulso comunitario para la promoción de políticas de igualdad. La batería de criterios e indicadores que ofrecen tanto la Red de políticas de igualdad a los municipios españoles que congrega como el Instituto Europeo de la Igualdad (EIGE), suponen pequeños ejemplos de la armonización de la política de igualdad por parte de instancias supranacionales, mediante el establecimiento de parámetros de calidad de políticas de igualdad que se instauran a través del conocimiento experto.

En este contexto, la tarea de inyectarle un golpe de espíritu transformador a la interseccionalidad pasa por explorar y reinventar la relación entre la praxis interseccional y las instituciones. Un diálogo entre la institucionalización de la interseccionalidad y los desafíos que, en un contexto de gobernanza neoliberal, ya está atravesando las políticas de igualdad y mainstreaming de género (Bacchi y Eveline 2003, Squires 2007), podría contribuir a la experimentación de otras formas de hacer la interseccionalidad (Collins y Bilge 2016) que abracen su dimensión heurística, el compromiso epistemológico con la justicia social y el desafío a la domesticación desde sus core themes. Explorar estos caminos resulta tarea necesaria para evitar que la interseccionalidad, un "constructo de la justicia social" (Collins 2016: 66) trazado a partir de las trayectorias de diversos movimientos contestatarios, acabe muriendo de éxito. 


\section{Agradecimientos}

La autora agradece a Jone Martínez-Palacios y a la/os revisora/es anónima/os sus observaciones y propuestas de mejora a este texto. Esta nota de investigación forma parte de una investigación doctoral en curso financiada por la Universidad del País Vasco, en el marco de las ayudas a la contratación para la formación de personal investigador.

\section{Referencias Bibliográficas}

Bacchi, C. y J. Eveline. 2003. "Mainstreaming and neoliberalism: A contested relationship". Policy and Society 22(2): 98118. https://doi.org/10.1016/S1449-4035(03)70021-6

Baiocchi, G. y E. Ganuza. 2017. Popular Democracy. The Paradox of Participation. California: Stanford University Press.

Bilge, S. 2016. "Plaidoyer pour une intersectionnalité critique non blanchie." Pp. 75-102 en L'Intersectionnalité: enjeux théoriques et politiques, coordinado por F. Fassa, E. Lépinard y M. Roca i Escoda. Paris: La Dispute.

Collins, P. H. y S. Bilge. 2016. Intersectionality. Cambridge: Polity Press.

Collins, P. H. 2000. Black feminist thought: Knowledge, Conciusness, and the Politics of Empowerment. New York: Routledge.

Collins, P. H. 2016. "Lost in translation? Black feminism, intersectionnalité et justice sociale." Pp. 53-74 en L'Intersectionnalité: enjeux théoriques et politiques, coordinado por F. Fassa, E. Lépinard y M. Roca i Escoda. Paris: La Dispute.

Combahee River Collective. 1981. "A Black Feminist Statement." Pp. 210-218 en This Bridge Called my Back: Writtings by Radical Women of Color, editado por C. Moraga y G. Anzaldúa. New york: Kitchen Table, Women of Color Press.

Crenshaw, K. 1989. "Demarginalizing the Intersection of Raze and Sex: A Black Feminist Critique of Antidiscrimination Doctrine, Feminist Theory and Antiracist Politics." University of Chicago Legal Forum 1: 139-167.

Crenshaw, K. 2016. "Les «voyages de l'intersectionnalité»." Pp. 29-52 en L'Intersectionnalité: enjeux théoriques et politiques, coordinado por F. Fassa, E. Lépinard y M. Roca i Escoda. Paris: La Dispute.

Hancock, A. 2007. "When multiplication doesn't equal quick addition: Examining intersectionality as a research paradigm". Perspectives on Politics 5(1): 63-79. https:// doi.org/10.1017/S1537592707070065

Hankivsky, O. y R. Cormier. 2011. Intersectionality and public policy: Some lessons from existing models. Political Research Quarterly, 64(1): 217-229.

Kantola, J. y K. Nousiainen. 2009. "Institutionalizing Intersectionality in Europe." International Feminist Journal Of Politics 11 (4): 459-477. https://doi. org/10.1080/14616740903237426

\section{NOTAS}

[1] A través de la fórmula mujeres de color este texto traduce de forma literal la noción inglesa women of color. Es una forma de mantener el modo de autorreferenciarse que emplean las mujeres racializadas en la literatura anglosajona, en cuya crítica se inspiran las autoras que se presentan.

Krizsan, A., H. Skjeie y J. Squires. 2012. Institutionalizing intersectionality. The changing nature of european equality regimes. London: Palgrave Macmillan.

Lascoumes, P. y P. Le Gales. 2007. "Introduction: Understanding Public Policy through Its Instruments. From the Nature of Instruments to the Sociology of Public Policy Instrumentation." Governance: An International Journal of Policy, Administration, and Institutions 20 (1): 1-21. https://doi.org/10.1111/j.1468-0491.2007.00342.x

Lombardo, E. y M. Verloo. 2010. "La 'interseccionalidad del género con otras desigualdades en la política de la Unión Europea." Revista Española de Ciencia Política 23: 11-30.

Martínez-Palacios, J. y P. Martínez. 2017. "Justicia social, interseccionalidad y profundización democrática." Investigaciones Feministas 8 (1): 9-17. https://doi. org/10.5209/INFE.54827

McCall, L. 2005. "The complexity of intersectionality". Signs 30(3): 1771-1800. https://doi.org/10.1086/426800

Möser, C. 2016. "Intersectionnalité et genre. Au sujet de la critique féministe des conditions de production du savoir." Pp. 103-129 en L'Intersectionnalité: enjeux théoriques et politiques, coordinado por F. Fassa, E. Lépinard y M. Roca i Escoda. Paris: La Dispute.

Platero, R. 2012. “¿Son las políticas de igualdad de género permeables a los debates sobre la interseccionalidad? Una reflexión a partir del caso español". Revista del CLAD Reforma y Democracia 52: 135-172.

Roca i Escoda, M., F. Fassa y E. Lépinard. 2016. L'Intersectionnalité: enjeux théoriques et politiques. Paris: La Dispute.

Squires, J. 2007. The new politics of gender equality. Nueva York: Palgrave Macmillan.

Verloo, M. 2006. "Multiple Inequalities, Intersectionality and the European Union". European Journal of Women's Studies 13 (3): 211-228. https://doi. org/10.1177/1350506806065753

Walby, S. 2007. "Complexity theory, systems theory, and multiple intersecting social inequalities". Philosophy of the Social Sciences 37(4): 449-470. https://doi. org/10.1177/0048393107307663

Yuval-Davis, N. 2006. "Intersectionality and feminist politics". European Journal of Women's Studies 13(3): 193-209. https://doi.org/10.1177/1350506806065752

UXUE ZUGAZA GOIENETXEA es investigadora predoctoral y miembro del grupo de trabajo Feminismos y Democracia del grupo de investigación Parte Hartuz, en la Universidad del País Vasco. Graduada en Ciencias Políticas y Gestión Pública por la misma universidad y máster en Democracia y Gobierno por la Universidad Autónoma de Madrid. Analiza la institucionalización de la interseccionalidad en el contexto de las políticas de igualdad en el Estado español. 